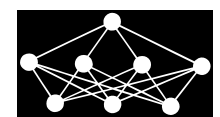

\title{
SOFT COMPUTING AND REGRESSION MODELLING APPROACHES FOR LINK-CAPACITY FUNCTIONS
}

\author{
C. Kosun*, G. Tayfur $\stackrel{\dagger}{\dagger}$ H.M. Celik
}

\begin{abstract}
Link-capacity functions are the relationships between the fundamental traffic variables like travel time and the flow rate. These relationships are important inputs to the capacity-restrained traffic assignment models. This study investigates the prediction of travel time as a function of several variables $V / C$ (flow rate/capacity), retail activity, parking, number of bus stops and link type. For this purpose, the necessary data collected in Izmir, Turkey are employed by Artificial Neural Networks (ANNs) and Regression-based models of multiple linear regression (MLR) and multiple non-linear regression (MNLR). In ANNs modelling, $70 \%$ of the whole dataset is randomly selected for the training, whereas the rest is utilized in testing the model. Similarly, the same training dataset is employed in obtaining the optimal values of the coefficients of the regression-based models. Although all of the variables are used in the input vector of the models to predict the travel time, the most significant independent variables are found to be $V / C$ and retail activity. By considering these two significant input variables, ANNs predicted the travel time with the correlation coefficient $R=0.87$ while this value was almost 0.60 for the regression-based models.
\end{abstract}

Key words: artificial neural networks, link-capacity functions, regression analysis, travel time, flow rate, capacity

Received: September 25, 2014

DOI: $10.14311 / \mathrm{NNW} .2016 .26 .007$

Revised and accepted: March 22, 2016

\section{Introduction}

The link-capacity could be basically described as the maximum number of vehicles that can reasonably be expected to traverse a specified location on a link within a time period [15, 18]. The capacity is an important parameter indicating performance of a link and it is utilized in link-capacity functions. The linkcapacity functions are the relationships between travel time and flow rate. The

\footnotetext{
* Caglar Kosun - Corresponding author, Department of City and Regional Planning, Izmir Institute of Technology, Gulbahce Campus, Urla, Izmir, 35430, Turkey, E-mail: cglrksn@gmail.com

${ }^{\dagger}$ Gokmen Tayfur, Department of Civil Engineering, Izmir Institute of Technology, Gulbahce Campus, Urla, Izmir, 35430, Turkey, E-mail: gokmentayfur@iyte.edu.tr

${ }^{\ddagger}$ H. Murat Celik, Department of City and Regional Planning, Istanbul Technical University, Taskisla Campus, Beyoglu, Istanbul, 34437, Turkey, E-mail: celikhus@itu.edu.tr
} 
link-capacity functions, i.e. link performance functions, are important elements of capacity-restraint traffic assignment process in urban travel demand modelling. Basically, the link-capacity functions are utilized to update travel times depending on the assigned flow rate at a selected link on transportation networks. Gastaldi and Rossi [7] state that the imprecise and unrealistic travel time-flow functions can cause failure in the improvement of transport models.

By default, the link-capacity functions embody one independent variable $(V / C)$ to determine the value of the dependent variable which is the travel time. By considering these two variables, several studies are employed to develop link-capacity functions [1]. According to the work of [1], for example, in 1964 Bureau of Public Roads (BPR) has proposed a link-capacity function that is widely used in traffic assignment studies. Moreover, in 1967 Overgaard has developed an alternative functional form for the link-capacity functions. However, there would be other variable(s) to be considered in link-capacity functions since traffic flow faces a number of interruptions on the transportation networks. Especially, apart from widely known traffic variables (e.g. flow rate and capacity), a number of urban planning decisions influence the travel time. At urban collectors, the factors such as the shopping, bus stops and parking along the roadway could affect the travel time and generate traffic congestion. This happens due to the blockage of at least one of the lanes, e.g., the violation of the right lanes by passenger buses, the illegal parking of vehicles and the loading and unloading of the goods.

In urban areas, travel time can be highly affected by those factors and it would be integrated with them. To the best knowledge of authors, there has been no considerable attempt to develop and express the link-capacity function within this perspective, which would also consider such variables/factors. This study intends to fill this gap by employing ANNs and regression-based modelling to predict the travel time. Although there is an extensive literature including ANNs-based [4, $5,14,20]$ and regression-based $[2,21]$ modelling on predicting traffic conditions, application of both modelling techniques, especially the ANNs, in the area of linkcapacity functions is not common.

This paper is organized as follows. In the next part, the general formulations of the link- capacity functions are presented. Afterwards, the necessary data, their collection methodologies and formulations are basically explained. Sections 4-7 focus on the ANNs and regression modelling and the results. In the last part, we conclude the paper and highlight our recommendations for future studies.

\section{An overview of link-capacity functions}

Developing link-capacity functions is quite important in transportation planning studies. In fact, [16] points out that it is impossible to model driver's route choice behaviour without a suitable link-capacity function for a transportation link or link groups. Moreover, the traffic assignment modelling that takes into account traffic congestion effect needs the mathematical relations embracing some traffic variables such as travel time, and capacity [13]. These relations can define the link-capacity functions or travel time functions of a network.

Branston [1] defines link-capacity functions within two approaches. The first mathematical approach provides simple relationship between travel time and traffic 
flow rate on a link that may be delicate for real data due to their simplicity. The widely preferable link-capacity function proposed by BPR is

$$
T=T_{0}\left(1+\alpha(V / C)^{\beta}\right),
$$

where $T$ is travel time, $T_{0}$ is travel time at zero flow, $V$ is traffic flow rate on a link, $C$ is capacity of a link, $\alpha$ and $\beta$ represent BPR-model parameters whose values are proposed as 0.15 and 4.0 , respectively $[11,12]$.

The second approach is theoretical and more complicated [1]. Link characteristics such as link widths can be embodied in this approach. Branston [1] shows several functions in this approach. For example

$$
T=\frac{t_{r}+t_{q}}{D}=\frac{1}{V_{r}}+\frac{t_{q}}{D}
$$

where $t_{r}$ is running travel time, $t_{q}$ is queuing travel time, $D$ is length of a link, $V_{r}$ is running speed.

In this study, we investigate other possible variables, besides $V / C$, such as retail activity, the number of bus stops, link type, parking that affect the travel time. These variables are not only specific in traffic engineering studies, but also they are very crucial in terms of urban transportation issues.

\section{Data collection and methodology}

For this study, bidirectional ten links were selected from the urban centre of Izmir which is located on the west part of Turkey. Six of these links are divided, whereas the rest are undivided (Tab. I). Furthermore, the divided links have two lanes in each direction while the undivided links have one lane in each direction. The lengths of the selected links are in range between $1 \mathrm{~km}$ and $3 \mathrm{~km}$ (Tab. I). The capacity of each of these links is computed by following the common procedure [15]

$$
C=s\left(\frac{g}{c_{y}}\right),
$$

where $C$ is capacity (vehicle per hour), $s$ is total saturation flow rate (vehicle per green hour), $g$ is effective green signal time (seconds), $c_{y}$ is signal cycle length (seconds).

From the traffic lights, the signal times are obtained for each route. To calculate capacity $(C)$ via Eq. (1), the effective green signal time (seconds) and signal cycle length (seconds) were utilized.

Total saturation flow rate is defined as follows [15]:

$$
s=s_{0} N f_{w} f_{h} f_{g} f_{p c} f_{b b} f_{a} f_{r t} f_{l t},
$$

where $s_{0}$ is ideal saturation flow rate per lane, usually taken to be 1900 passenger car per green hour per lane, $N$ is number of lanes in direction, $f_{w}$ is adjustment factor for lane width, $f_{h}$ is adjustment factor for heavy vehicle presence, $f_{g}$ is adjustment factor for grade, $f_{p c}$ is adjustment factor for parking conditions, $f_{b b}$ is adjustment factor for local bus blockage, $f_{a}$ is adjustment factor for area type, $f_{r t}$ is 
adjustment factor for right-turning vehicles, $f_{l t}$ is adjustment factor for left-turning vehicles.

In this study, only ideal saturation flow rate $\left(s_{0}\right)$ and number of lanes $(N)$ for each direction are performed to calculate the total saturation flow rate $(s)$.

\begin{tabular}{lcccc}
\hline Link name & $\begin{array}{c}\text { Link } \\
\text { length } \\
{[\mathrm{km}]}\end{array}$ & Link type & $\begin{array}{c}\text { The number } \\
\text { of main } \\
\text { intersections }\end{array}$ & Location \\
\hline Anadolu street & 1.7 & Undivided & 4 & Urban Centre \\
Erdoğan Akkaya street & 1.6 & Undivided & 6 & Urban Centre \\
Mithatpaşa (Karataş) street & 1.3 & Undivided & 5 & Urban Centre \\
Mithatpaşa (Göztepe) street & 2.9 & Undivided & 10 & Urban Centre \\
Girne street & 1.9 & Divided & 10 & Urban Centre \\
Inönü street & 1.6 & Divided & 4 & Urban Centre \\
Şair Eşref street & 1.5 & Divided & 6 & Urban Centre \\
Talatpaşa street & 1.2 & Divided & 5 & Urban Centre \\
Mithatpaşa street & 2.2 & Divided & 6 & Urban Centre \\
Mustafa Kemal street & 1.75 & Divided & 6 & Urban Centre \\
\hline
\end{tabular}

Tab. I General descriptions of the selected links.

There are some necessary data to be collected before the modelling process. The data on the directional travel time through the links, directional volume, signal cycle lengths [6] (to calculate capacity), the number of lanes, the number of the bus stops at each direction, the presence of parking through the links, the presence of retail activity along the links, and link type data were collected for each link. The last three independent variables are discrete, whereas the others are continuous variables. Several descriptive statistics of the continuous variables are summarized in Tab. II.

\begin{tabular}{lcccc}
\hline Variable name & Minimum & Maximum & Mean & $\begin{array}{c}\text { Standard } \\
\text { deviation }\end{array}$ \\
\hline T_TIME (output) [s/km] & 94.1 & 297.3 & 153.1 & 45.8 \\
$V / C$ ratio (input) & 0.12 & 0.97 & 0.51 & 0.18 \\
The number of bus stops & & & & \\
per km (input) & 0 & 3.75 & 2.65 & 1.12 \\
Volume [veh/hr] & 139 & 1251 & 595 & 294 \\
Capacity [veh/hr] & 475 & 3800 & 1495 & 760 \\
\hline
\end{tabular}

Tab. II Descriptive statistics of the continuous variables.

The data collection was performed on the specified weekdays within June-July 2009 on the selected links at the peak hour periods. The peak-hour periods for the links are 08:00-09:00 a.m., 12:00-13:00 p.m. and 18:00-19:00 p.m. The travel time data collection at the links is performed simultaneously by running test car through 
the links and recorded manually by using stopwatch. The traffic volume and vehicle length data in every five minutes' period are collected by portable traffic counters. Thus, a total of 84 datasets were collected.

The flow rate for each direction is obtained by

$$
V=\frac{V_{0}}{P H F f_{H V} f_{P} N},
$$

where $V$ is equivalent flow rate during peak 15 minutes (passenger car per hour), $V_{0}$ is hourly volume (vehicle per hour), $P H F$ is peak hour factor, $f_{H V}$ is adjustment factor for heavy vehicles in the traffic stream, $f_{p}$ is adjustment factor for driver population, and $N$ is the number of lanes [15].

Eq. (2) provides a conversion of the hourly volume into the flow rate that refers to the hourly passenger car equivalent flow rate. Since in this study there are no data related to the adjustment factor $\left(f_{p}\right)$ for driver population, its value is taken as 1 that is a default value presented in Highway Capacity Manual [18]. Adjustment factor $\left(f_{H V}\right)$ for heavy vehicles in the traffic stream is calculated as

$$
f_{H V}=\frac{1}{1+P_{T}\left(E_{T}-1\right)+P_{R}\left(E_{R}-1\right)},
$$

where $P_{T}$ is proportion of trucks and buses in the traffic stream, $E_{T}$ is passenger car equivalent for trucks and buses, $P_{R}$ is proportion of recreational vehicles in the traffic stream, and $E_{R}$ is passenger car equivalent for recreational vehicles [18].

The value of $P_{R}$ becomes zero (0) owing to a lack of $P_{R}$ data for this study. Additionally, $E_{T}$ is taken as 1.5 that is proposed in Highway Capacity Manual [18]. Lastly, $P_{T}$ value is computed by taking into account the observed vehicle lengths. In this study, it is assumed that the vehicles that are at least 8.5 meters length are considered as the heavy vehicles. For 15 minutes period, we calculate the peak hour factor $(P H F)$ by

$$
P H F=\frac{V_{0}}{4 V_{15}},
$$

where $V_{15}$ is maximum 15-minute volume within the hour (vehicle) [15].

\section{Artificial neural networks (ANNs) modelling}

ANNs have an ability to identify patterns between input and output variables. In the commonly employed three-layer feed-forward neural network (FFNN), the input quantities are fed into the input layer neurons which, in turn, pass them on to the hidden layer neurons after multiplying them by the connection weights. A hidden layer neuron adds up the weighted input received from each input neuron, associates it with a bias, and then passes the result $\left(\right.$ net $\left._{j}\right)$ on through the activation (transfer) function, which can be sigmoid or tangent hyperbolic [8].

Similarly, the produced outputs from the inner neurons are passed to the network output neuron. The net information received by the output neuron from the inner neurons is passed through the activation function to produce the network output. The optimal weights are found by minimizing a predetermined error function $(E)$ of the following form $[17,19]$ 


$$
E=\sum_{P} \sum_{p}\left(y_{i}-t_{i}\right)^{2},
$$

where $y_{i}$ is the component of an ANNs output vector $Y, t_{i}$ is the component of a target output vector $T, p$ is the number of output neurons, and $P$ is the number of training patterns.

The gradient-descent method, along with the chain rule of differentiation, is generally employed to modify the network weights as $[8,17]$

$$
\Delta v_{i j}(n)=-\delta \frac{\partial E}{\partial v_{i j}}+\eta \Delta v_{i j}(n-1),
$$

where $\Delta v_{i j}(n)$ and $\Delta v_{i j}(n-1)$ are the weight increments between node $i$ and $j$ during the $n$-th and $(n-1)$-th pass or epoch, $\delta$ is the learning rate, and $\eta$ is the momentum factor. The details of ANNs can be obtained from $[8,17,19]$.

In this study, three-layer FFNN is constructed. $V / C$, parking, retail activity, bus stops, and link type variables formed the input vector while the travel time was the output variable. Thus, the network had 5 input neurons and 1 output neuron. The number of neurons in the hidden layer was obtained as 4 by the trial and error procedure. Note that three layer neural network (i.e., the network having single hidden layer) is commonly employed in the literature [3, 10, 14, among others]. For very complex problems of having many input variables, multiple output variables and excessive number of datasets multi-hidden layer neural networks may be employed.

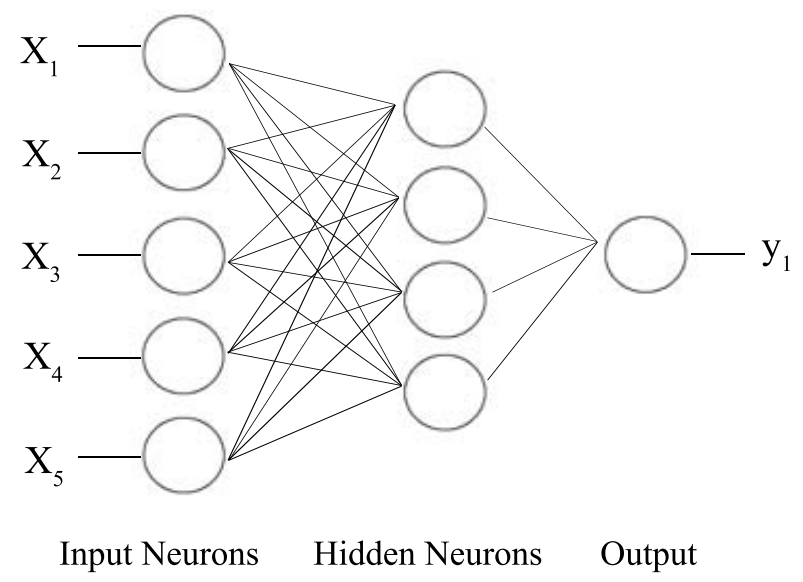

Fig. 1 An illustration of three-layer feed-forward ANNs model.

The collected 84 dataset is randomized and then divided in two parts for the ANNs modelling. $70 \%$ of whole data was used for the training stage, whereas the remaining data was used for testing the network. The randomized datasets for each variable are subject to $t$-test. As seen in Tab. III, the computed $t$-values do not fall into the critical region (i.e., $t=+1.96$ and -1.96 ), thus satisfying the $t$-test. 
Kosun C., Tayfur G., Celik H.M.: Soft computing and regression...

\begin{tabular}{lccccc}
\hline Variables & Training dataset & \multicolumn{2}{c}{ Testing dataset } & $t$-value \\
\hline & Mean & $\begin{array}{c}\text { Standard } \\
\text { deviation }\end{array}$ & Mean & $\begin{array}{c}\text { Standard } \\
\text { deviation }\end{array}$ & $\begin{array}{c}\text { significance } \\
\text { level }=0.05\end{array}$ \\
\hline T_TIME $[\mathrm{s} / \mathrm{km}]$ & 152.1 & 48.49 & 155.4 & 39.9 & -0.3 \\
$V / C$ & 0.51 & 0.19 & 0.53 & 0.18 & -0.45 \\
BUSSTOPS $[1 / \mathrm{km}]$ & 2.7 & 1.09 & 2.6 & 1.2 & -0.48 \\
\hline
\end{tabular}

Tab. III Indicators of training and testing dataset for the variables.

The hyperbolic tangent activation function is calculated as

$$
f\left(\text { net }_{j}\right)=\frac{\mathrm{e}^{\text {net }_{j}} \mathrm{enet}_{j}}{\mathrm{e}^{\text {net }_{j}}+\mathrm{e}^{\text {net }_{j}}} .
$$

Eq. (3) is employed in the training of the network. The network was trained with 1000 iterations, learning rate of 0.1 and momentum factor of 0.5. In this study, NeuroSolutions 5.0 package program was used for the ANNs modelling.

\section{Regression modelling}

Regression analysis is applicable to many subjects in a wide variety of disciplines. The performance of regression modelling depends on the explanation of total variance in data. Its main goal is to minimize sum of squares of the differences between observed and estimated values of a variable by the least squares method. MLR model, in a general form, can be expressed by

$$
y=b_{0}+b_{1} x_{1}+b_{2} x_{2}+\cdots+b_{i} x_{i}+\varepsilon,
$$

where $y$ is dependent (output) variable, $x_{i}$ are independent (input) variables, $b_{i}$ are linear regression-model coefficients, and $\varepsilon$ is error term.

In this study, the following MLR model is constructed with variables flow rate/capacity ratio $(V / C)$ and retail activity (RETAIL) as follows:

$$
\text { T_TIME }=b_{0}+b_{1}(V / C)+b_{2}(\text { RETAIL }) .
$$

As discussed in Section 6, the sensitivity analysis by ANNs showed that $V / C$ and RETAIL are the most significant input parameters. Hence, in Eq. (4), only these variables are considered. In a similar fashion, MNLR model is constructed as follows:

$$
\text { T_TIME }=a(V / C)^{b}+c(\text { RETAIL })^{d},
$$

where $a, b, c$ and $d$ are non-linear regression-model coefficients.

By employing the calibration data, which are used for training the ANNs, were employed to find the optimal values of the coefficients of the regression-based models. 


\section{ANNs modelling results and discussion}

In the ANNs modelling, the parking, number of bus stops (per km), retail activity, link type, and $V / C$ ratio formed the input variables, whereas the observed travel time was the output variable. We assigned 0 and 1 for link type ( 0 for undivided links, and 1 for divided links), parking and retail activity ( 0 for non-existence, and 1 for existence).

Fig. 2 and Fig. 3 present measured travel time versus ANNs-predicted results for the testing stage. As seen in Fig. 2, the model closely predicts the measured data with low mean absolute error, $\mathrm{MAE}=14$, high $R=0.90$ which is a statistical measure assessing how well the observed data are matched by the model results. The high value of $R$ indicates that this match is satisfactory.

As seen in Fig. 3, all the data stays inside the error bounds [17]. The distribution along the fitting line is symmetrical, implying that the model neither over predicts nor under predicts the data.

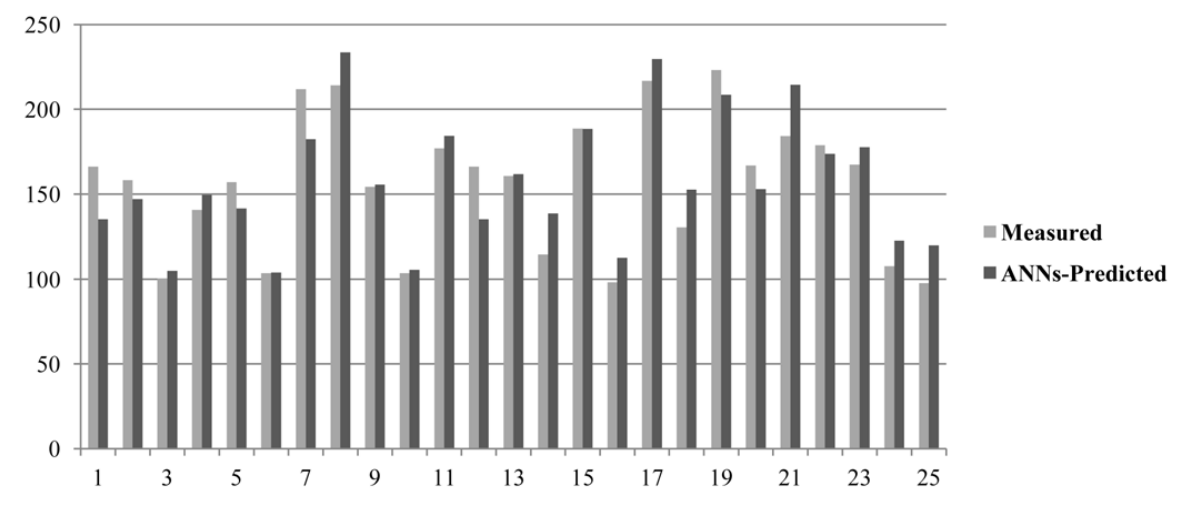

Fig. 2 Comparison of measured travel time and ANNs-predicted travel time (testing stage).

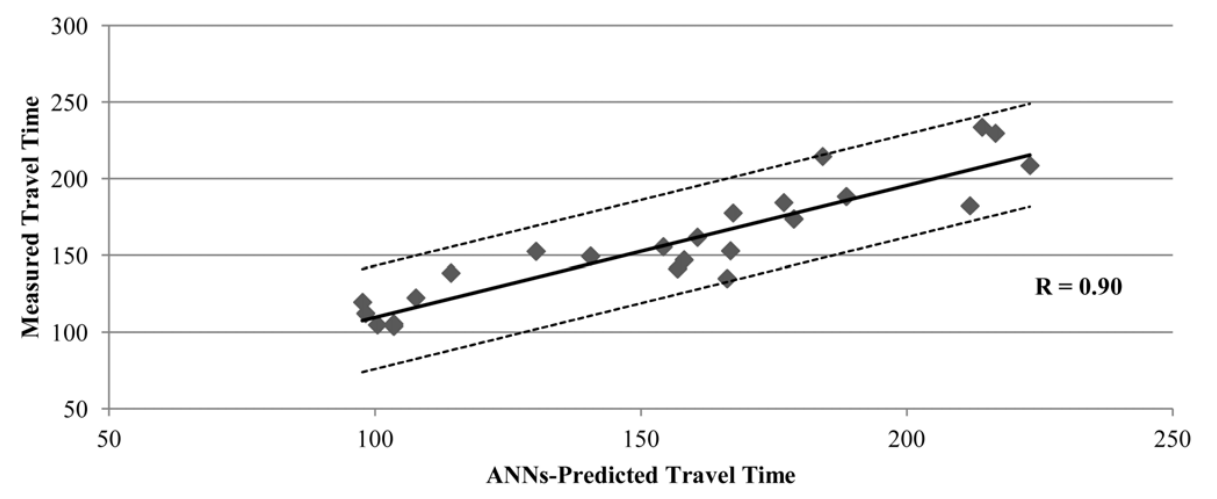

Fig. 3 Comparison of measured versus ANNs-predicted results (testing stage) of travel time. 
The next step is to understand the significance of each variable. Thus, each of the variables is added in and omitted from the modelling process. Therefore, depending on the changing modelling results due to variable inclusion and omission, the significance of the each variable is obtained. The sensitivity analysis results are summarized in Tab. IV.

\begin{tabular}{ccccc}
\hline Model & MAE & $R$ & Model inputs & Model output \\
\hline 1 & 14 & 0.90 & $V / C$, PARKING, BUSSTOPS, RETAIL, LINK_TYPE & T_TIME \\
2 & 20 & 0.77 & PARKING, BUSSTOPS, RETAIL, LINK_TYPE & T_TIME \\
3 & 15 & 0.87 & $V / C$, BUSSTOPS, RETAIL, LINK_TYPE & T_TIME \\
4 & 13 & 0.91 & $V / C$, PARKING, BUSSTOPS, RETAIL & T_TIME \\
5 & 21 & 0.75 & $V / C$, PARKING, BUSSTOPS, LINK_TYPE & T_TIME \\
6 & 14 & 0.90 & $V / C$, PARKING, RETAIL, LINK_TYPE & T_TIME \\
7 & 16 & 0.87 & $V / C$, RETAIL & T_TIME \\
\hline
\end{tabular}

Tab. IV Sensitivity analysis results showing inputs' contribution in the ANNs modelling.

According to Tab. IV, when the variable bus stop is omitted from the modelling, the model results do not change. On the other hand, when the variable bus stop is added and retail activity variable is omitted from the input vector the results change considerably. Similarly, when the variable $V / C$ ratio is solely excluded from the input vector, the modelling results change significantly. These results (in Tab. IV) indicate that the variables $V / C$ ratio and retail activity are the most significant inputs. The least significant inputs, according to Tab. IV, are found to be the bus stops, parking, and the link type. In other words, as seen in Tab. IV, Model 7 with two input parameters (input variables are $V / C$ and RETAIL) produce quite comparable results to the other models (Models $1,3,4$ and 6 in Tab. IV) which employ 4 or 5 inputs.

Fig. 4 presents measured versus predicted travel time for the testing stage of the neural networks employing only $V / C$ and RETAIL in the input vector (Model 7).

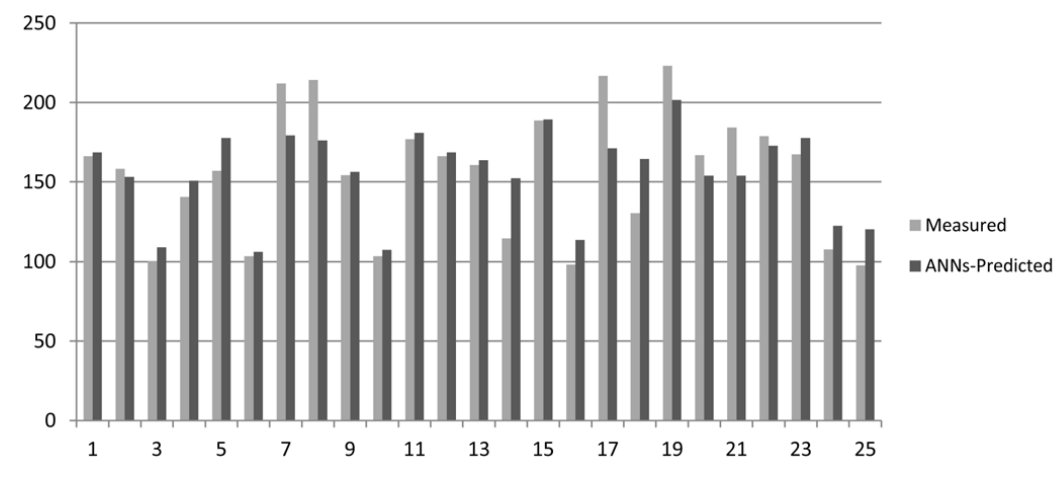

Fig. 4 Comparison of measured travel time and ANNs-predicted travel time (testing stage). 
As seen in Fig. 4, the model closely predicts the measured data with low MAE = 16 , high $R=0.87$. All the data remains inside the error bounds (Fig. 5). The distribution along the fitting line is symmetrical, implying that the model neither over predicts nor under predicts the data (Fig. 5).

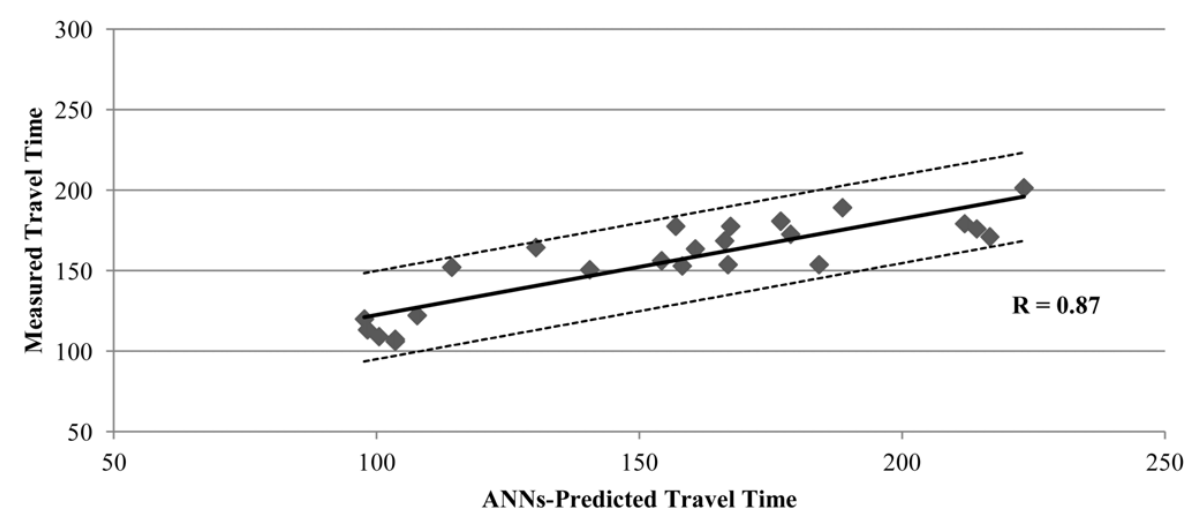

Fig. 5 Comparison of measured versus ANNs-predicted results (testing stage) of travel time.

\section{Regression modelling results and discussion}

The variables $V / C$ and retail activity are employed in regression models as the independent variables Eq. (4) and Eq. (5). Performing the calibration (the training data used in ANNs) data, the optimal values of the coefficients of Eq. (4) and Eq. (5) are obtained. Thus, the resulting MLR and MNLR models are as follows:

$$
\begin{aligned}
& \mathrm{T}_{\text {_TIME }}=87.8+54.9(V / C)+51.9(\mathrm{RETAIL}), \\
& \mathrm{T}_{\text {_TIME }}=137.3(V / C)^{0.22}+50.4(\mathrm{RETAIL})^{2.5} .
\end{aligned}
$$

The coefficients of the regression equations were obtained by using the method of least squares. We used the statistical package program of SPSS [9].

To test the performances of the regression models, we applied them to the validation (testing) dataset. We obtained $R$ values of 0.62 , and 0.60 with MAE of 17 and 18 for MLR and MNLR models, respectively.

\section{Conclusions}

This study predicts the travel time with respect to $V / C$ and the other aforementioned variables for the selected links in the urban area of Izmir. The prediction modelling is based on ANNs, MLR and MNLR. It is proved that the retail activity and $V / C$ variables are important in the link-capacity functions, therefore, travel time would be updated more sensitively. One of the main contributions of this study is to show the use of $V / C$ and RETAIL together as input parameters 
in the link-capacity functions. Other important contribution of this study is the employment and comparative application of ANNs and regression-based models in the link-capacity functions.

The following conclusions are drawn from this study:

1. ANNs modelling technique seems more explanatory than regression-based modelling in terms of contributions of independent variables in the modelling process. It is apparent that the ANNs performance is higher than the regression models for this study.

2. The presence of retail activity along urban links can lead to increase in travel time. To decrease possible traffic delays on transportation network, this criterion would be considered in urban traffic studies as well.

3. BPR-type equation involving retail activity variable may be developed in future studies.

4. In addition, other land-use decisions especially around the urban collectors would be taken into account in a broader perspective and integrated with link-capacity functions in future case studies.

5. Developing link-capacity functions can avoid the dependency on fixed BPR model parameters in urban transportation demand modelling.

6. Note that this study deals with travel times for the urban collectors rather than highways and freeways.

\section{References}

[1] BRANSTON D. Link capacity functions: A review. Transportation Research. 1976, 10(4), pp. 223-236, doi: 10.1016/0041-1647(76)90055-1.

[2] CLARK S. Traffic prediction using multivariate nonparametric regression. Journal of Transportation Engineering. 2003, 129(2), pp. 161-168, doi: 10.1061/(ASCE) 0733-947X (2003) $129: 2(161)$.

[3] DHARIA A., ADELI H. Neural network model for rapid forecasting of freeway link travel time. Engineering Applications of Artificial Intelligence. 2003, 16(7-8), pp. 607-613, doi: 10.1016/j.engappai.2003.09.011.

[4] DIA H. An object-oriented neural network approach to short-term traffic forecasting. European Journal of Operational Research. 2001, 131(2), pp. 253-261, doi: 10.1016/ S0377-2217(00)00125-9.

[5] DOUGHERTY M.S., COBBETT M.R. Short-term inter-urban traffic forecasts using neural networks. International Journal of Forecasting. 1997, 13(1), pp. 21-31, doi: 10.1016/ S0169-2070 (96) 00697-8.

[6] D.T.C. Traffic Signalization Data. Izmir Metropolitan Municipality, Department of Traffic and Control, Izmir, Turkey, 2009.

[7] GASTALDI M., ROSSI R. A methodology for calibrating road link travel time functions using data from driving simulator experiments. Procedia-Social and Behavioral Sciences. 2011, 20, pp. 656-665, doi: 10.1016/j.sbspro.2011.08.073.

[8] HAYKIN S. Neural Networks: A Comprehensive Foundation. New Jersey: Prentice-Hall, 1999. 


\section{Neural Network World 2/2016, 129-140}

[9] IBM SPSS Regression 20 Manual [online]. IBM Corporation, U.S., 2011 [viewed 2016-04-17]. Available from: ftp://public.dhe.ibm.com/software/analytics/spss/documentation/ statistics/20.0/en/client/Manuals/IBM_SPSS_Regression.pdf

[10] JEONG R., RILETT L.R. Bus arrival time prediction using artificial neural network model. In: Proceedings of the 7th International IEEE Conference on Intelligent Transportation Systems. IEEE, 2004, pp. 988-993, doi: 10.1109/ITSC.2004.1399041.

[11] OPPENHEIM N. Urban Travel Demand Modeling: From Individual Choices to General Equilibrium. New York: John Wiley \& Sons, 1995.

[12] ORTIGOSA J., MENENDEZ M. Traffic performance on quasi-grid urban structures. Cities 2014, 36, pp. 18-27, doi: 10.1016/j.cities.2013.08.006.

[13] ORTUZAR J.D., WILLUMSEN L.G. Modelling Transport. Chichester: John Wiley \& Sons, 2001.

[14] PARK D., RILETT L.R. Forecasting freeway link travel times with a multilayer feedforward neural network. Computer-Aided Civil and Infrastructure Engineering. 1999, 14(5), pp. 357-367, doi: 10.1111/0885-9507.00154

[15] ROESS R.P., MCSHANE W.R., PRASSAS E.S. Traffic Engineering. New Jersey: Prentice-Hall, 1998.

[16] SUH S., PARK C.H., KIM T.J. A highway capacity function in Korea: Measurement and calibration. Transportation Research Part A: General. 1990, 24(3), pp. 177-186, doi: 10. 1016/0191-2607(90)90055-B.

[17] TAYFUR G. Soft Computing in Water Resources Engineering: Artifical Neural Networks, Fuzzy Logic and Genetic Algorithms. Southampton: WIT Press, 2012.

[18] T.R.B. Highway Capacity Manual. Washington, DC: Transportation Research Board, 2000.

[19] TSOUKALAS L.H., UHRIG R.E. Fuzzy and Neural Approaches in Engineering. New York: John Wiley \& Sons, 1997.

[20] VAN LINT J.W.C. Reliable real-time framework for short-term freeway travel time prediction. Journal of Transportation Engineering. 2006, 132(12), pp. 921-932, doi: 10.1061/ (ASCE) 0733-947X (2006) 132:12(921).

[21] ZHANG X., RICE J.A. Short-term travel time prediction. Transportation Research Part C: Emerging Technologies. 2003, 11(3-4), pp. 187-210, doi: 10.1016/S0968-090X (03)00026-3. 